Association for Information Systems AIS Electronic Library (AISeL)

Wirtschaftsinformatik Proceedings 2001

Wirtschaftsinformatik

September 2001

\title{
Die drei Bereiche des kontinuierlichen Anforderungsmanagements
}

Bruno Schienmann

Infomatikzentrum der Sparkassenorganisation (SIZ) GmbH, bruno.schienmann@siz.de

Klaus Pohl

Universität Essen, pohl@cs.uni-essen.de

Follow this and additional works at: http://aisel.aisnet.org/wi2001

\section{Recommended Citation}

Schienmann, Bruno and Pohl, Klaus, "Die drei Bereiche des kontinuierlichen Anforderungsmanagements" (2001). Wirtschaftsinformatik Proceedings 2001. 45.

http://aisel.aisnet.org/wi2001/45

This material is brought to you by the Wirtschaftsinformatik at AIS Electronic Library (AISeL). It has been accepted for inclusion in Wirtschaftsinformatik Proceedings 2001 by an authorized administrator of AIS Electronic Library (AISeL). For more information, please contact elibrary@aisnet.org. 
In: Buhl, Hans Ulrich, u.a. (Hg.) 2001. Information Age Economy; 5. Internationale Tagung Wirtschaftsinformatik 2001. Heidelberg: Physica-Verlag

ISBN: 3-7908-1427-X

(C) Physica-Verlag Heidelberg 2001 


\title{
Die drei Bereiche des kontinuierlichen Anforderungsmanagements
}

\author{
Bruno Schienmann \\ Infomatikzentrum der Sparkassenorganisation (SIZ) GmbH
}

Klaus Pohl

Universität Essen

Anstelle einer Zusammenfassung.:

»Unsere Anforderungen bleiben während eines Entwicklungsprojektes weitgehend stabil, da wir die eigentlichen Kundenprobleme systematisch ermittelt und priorisiert haben und frühzeitig eine klare Produktvision und-abgrenzung existierte. Natürlich gibt es auch Änderungen. Diese werden in einem kontrollierten, für alle Beteiligten transparenten Prozess in die Umsetzung geführt. Das Projektteam kann sich auf die Detaillierung und Umsetzung der Anforderungen konzentrieren. Es existieren klare Auftraggeber-/Auftragnehmer-Strukturen zwischen Kunden, Produktmanagern und Entwicklung. Da wir ein kontinuierliches Risiko- und Umsetzungsmanagement verfolgen, kennen wir jeweils den aktuellen Projektstatus und können aktiv Problemen entgegenwirken. Das Verhältnis zwischen Entwicklung, Kunden und Anwendern ist gut, alle Gruppen arbeiten eng zusammen." (ein zufriedener IT-Leiter)

Schlüsselworte: Requirements Engineering, Requirements Management, kontinuierliches Anforderungsmanagement

\section{Motivation und Übersicht}

Schöne neue Welt? Die obige, fiktive Feststellung eines IT-Leiters entspricht wohl nicht den Erfahrungen der meisten Leser. Entwicklungen und Praxiserfahrungen in den letzten Jahren zeigen jedoch, dass durch ein systematisch betriebenes Anforderungsmanagement das einleitend skizzierte Szenario keine Vision bleiben muss. Bewährte Methoden, Techniken und Werkzeuge stehen bereit, um dieses Ziel eines effektiven Anforderungsmanagements schrittweise zu verwirklichen. Im letzten Jahr wurde im SIZ in einem Grundlagenprojekt ein umfassender Leitfaden zum Thema Anforderungsmanagement erarbeitet. Die Initiierung dieses Projektes entstand im Rahmen von Diskussionen und Workshops um die Verwaltung und 
Umsetzung von Anforderungen in Groß-Projekten und in Entwicklungseinheiten der Sparkassen-Finanzgruppe (vgl. etwa [Kräft99]).

In diesem Beitrag sollen die wesentlichen Inhalte und Kernideen des Leitfadens bezogen auf die verfolgten Ziele eines kontinuierlichen Anforderungsmanagements (vgl. dazu [Pohl99]) vorgestellt werden. Erste Erfahrungen bei der Nutzung dieses Leitfadens in einem Großprojekt zur Entwicklung eines Kernbanksystems beschreibt [Schienmann00a], eine Übersicht des Leitfadens mit Aktivitäten, Ergebnistypen, Techniken und Best Practices gibt [Schienmann00b].

\section{Herausforderungen}

Betrachtet man die gegenwärtige Praxis, so ist das Anforderungsmanagement oft unbefriedigend gelöst. Das Vorgehen ist häufig wenig strukturiert. Rollen und Kompetenzen werden nach Gewohnheit eingenommen, Planungen, Ergebnisse, und Entscheidungen sind kaum nachvollziehbar. Anforderungen konsistent und stabil zu verwalten verursacht oft große Mühe. Liegt die Ursache für die häufigen Änderungen wirklich in den vielen Änderungen bzgl. der Anforderungen oder nicht vielmehr in der oberflächlichen Anforderungserhebung und -validierung?

Untersuchungen etwa der Standish Group [Standish95] oder European Software Process Improvement Training Initiative [ESPITI95] zeigen, dass mehr als die Hälfte aller Anwendungsentwicklungsprojekte die Projektlaufzeit weit überziehen und etwa 1/3 aller Projekte ergebnislos ganz abgebrochen werden. Als Ursache für diese Abweichungen stehen Fehler, die auf fehlendes oder fehlerhaftes Anforderungsmanagement zurückgehen, mit etwa $40 \%$ an erster Stelle. Umgekehrt gilt nach Erhebungen der Standish Group: ,...managing requirements well was the factor most related to successful projects".

Inzwischen existieren eine Reihe fundierter Arbeiten aus Forschungsprojekten wie NATURE oder CREWS sowie gute praxisnahe Literatur zum Requirements Engineering (vgl. bspw. [Davis93, Gause93; Pohl96; Robertson99; Wiegers99; Rupp01]). Das Thema Anforderungsmanagement wird dabei allerdings fast immer nur aus Projektsicht behandelt. Umfassendere Ansätze aus dem Systems Engineering wie [Martin96] oder [Stevens98] werden im Software Engineering bisher wenig reflektiert. Selbst neuere Literatur zu diesem Thema, welche explizit den Management-Aspekt hervorhebt (vgl. etwa das ansonsten empfehlenswerte Buch von Leffingwell und Widrig [Leffingwell99]) bieten nur ansatzweise einen übergreifenden, integrierten Ansatz für das Thema Anforderungsmanagement.

Sicherlich können bereits durch sehr einfache Maßnahmen, wie die frühzeitige Anforderungspriorisierung oder die Definition von Abnahmekriterien, gute Erfolge erzielt werden. Um jedoch grundlegende Verbesserungen im Umgang mit Anforderungen zu erreichen, genügt es nicht, einzelne gute und bewährte Techni- 
ken, wie Anwendungsfälle (use cases) für die Spezifikation von Anforderungen, Problem Frames für die Problemstrukturierung und Quality Function Deployment $(Q F D)$ für die Produktplanung einzuführen sowie einzelne organisatorische Maßnahmen wie etwa ein Change Control Board zu etablieren. Stattdessen muss ein projekt- und phasenübergreifendes Anforderungsmanagement als kontinuierlicher, permanenter Prozess von der Erhebung einer Kundenanforderung und Beauftragung bis zur Lösungsbereitstellung und anschließenden Kundenbetreuung in einer Organisation eingeführt und verankert werden. Ein solches kontinuierliches Anforderungsmanagement kann dann auch einen wesentlichen Beitrag zur erfolgreichen Veränderung von Organisationen über ein systematisch betriebenes Business Engineering leisten und die Konvergenz von Geschäft und IT vorantreiben.

\section{Kernidee und Vision}

Als eine der Sciences of the Artificial beschäftigt sich die Anwendungsentwicklung mit der Konstruktion künftiger künstlicher Gegenstände mit geplanten Eigenschaften. Diese künstlichen Gegenstände oder Artefakte dienen einem bestimmten Zweck, wobei zur Zweckerfüllung die Abstimmung der Beschaffenheit des Artefakts mit seinem Zweck und seiner Umwelt erforderlich ist: »Whether a knife will cut, depends on the material of its blade and the hardness of the substance to which it is applied $\ll[$ Simon85, S. 6].

Der Entwurf eines Anwendungssystems lässt sich in diesem Sinne als das zweckgerichtete planende Anpassen der Beschaffenheit dieses Systems an die Aufgabenstellungen des Anwendungsbereichs auffassen. Aufgabe und Zielsetzung des Anforderungsmanagements ist es, die aus diesen Aufgabenstellungen resultierenden Anforderungen der Kunden an das System oder IT-Produkt zu spezifizieren, diese kontinuierlich an Veränderungen des Anwendungsbereichs anzupassen und deren Umsetzung in Entwicklungsprojekten sicherzustellen. 


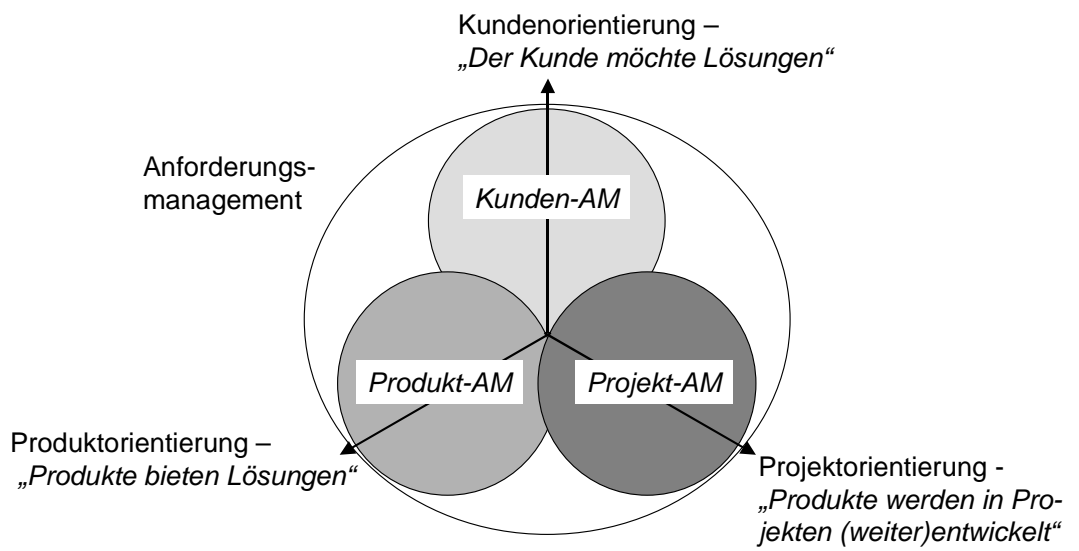

Abbildung 1: Sichten des Anforderungsmanagements

Die Kernidee des Leitfadens ist es, das Anforderungsmanagement aus den drei Sichten Kunde, Produkt und Projekt zu entfalten und damit den AM-Prozess durchgängig zu gestalten (vgl. Abbildung 1):

- Kundenorientierung. Kunden stellen Anforderungen, um Lösungen für ihre Probleme zu erhalten. Ihre Kundenanforderungen müssen sich weder auf konkrete Produkte noch Projekte beziehen.

- Produktorientierung. Produkte stellen Lösungen für diese Probleme bzw. die Anforderungen der Kunden dar. Produktanforderungen werden auf der Basis von Kundenanforderungen spezifiziert und in Projekten umgesetzt.

- Projektorientierung. In Anwendungsentwicklungsprojekten mit begrenzter Laufzeit und definierter Zielsetzung werden Produkte realisiert und damit Problemlösungen für den Kunden bereitgestellt.

Aus diesen drei Sichten leiten sich die zentralen Prozessbereiche eines kontinuierlichen, durchgängigen Anforderungsmanagements ab (Abbildung 2):

1. Kunden-Anforderungsmanagement (Kunden-AM): Das Kunden-AM stellt sicher, dass die Kundenbedürfnisse in der Systementwicklung berücksichtigt und in Produkte umgesetzt werden.

2. Produkt-Anforderungsmanagement (Produkt-AM): Das Produkt-AM sorgt für die Nachhaltigkeit der Produkt- bzw. Anwendungsentwicklung. Es überführt Kunden- in Produktanforderungen und bündelt diese zu Produktreleases. 
3. Projekt-Anforderungsmanagement (Projekt-AM): Das Projekt-AM detailliert die Produktanforderungen und setzt diese unter Einhaltung der gesetzten Rahmenbedingungen um.

Jeder dieser Bereiche beschäftigt sich unterschiedlich mit Anforderungen, mit speziellen Ausrichtungen, Methoden und Techniken. Ziele der Unterscheidung sind:

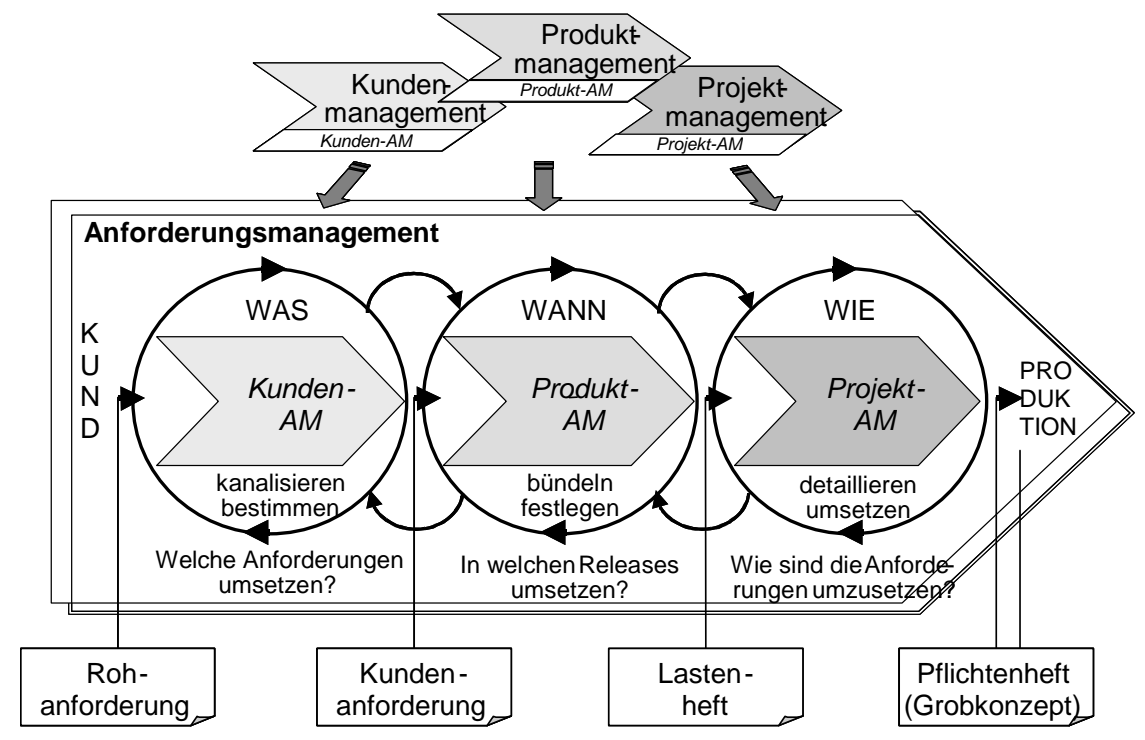

Abbildung 2: Prozessbereiche des Anforderungsmanagements

- Durch klare Aufgabengebiete eine Konzentration der Prozessverantwortlichen auf die Kernkompetenzen (Kunde, Produkt, Projekt) zu ermöglichen.

- Eindeutige Verantwortungsbereiche mit definierten Auftraggeber- und Auftragnehmerrollen ohne Rollenkonflikte festzulegen.

- Den Umgang mit Anforderungen für alle Beteiligten vom Kunden bis zur Produktion transparent zu machen.

Ein geeignetes Umfeld für die Umsetzung von Anforderungen auch in leichtgewichtigen, schnellen Anwendungsentwicklungsprozessen zu schaffen. Die Unterscheidung dieser Bereiche soll also nicht zusätzlichen Verwaltungsaufwand für die Anwendungsentwicklung erzeugen. Im Gegenteil, die Aufteilung der Verantwortlichkeiten und die kontinuierliche Wahrnehmung von Aufgaben im KundenAM und Produkt-AM schafft geeignete Rahmenbedingungen für leichtgewichtige Entwicklungsprojekte und schnelle Entwicklungszyklen, da frühzeitig kleine Produktreleases geschnitten, beauftragt und realisiert werden können.

Natürlich erfordert dies eine enge Zusammenarbeit und Koordination aller drei Bereiche. Das Produkt-AM hat hierbei eine zentrale Aufgabe: Es stellt die Nach- 
haltigkeit der Produkt(familien)entwicklung sicher und entzerrt bzw. synchronisiert das Kunden-AM (Fachseite) und das Projekt-AM (Entwicklung). Hierbei solle es zudem die typischen Probleme eines ausschließlich am Entwicklungsprojekt orientierten Umgangs mit Anforderungen verhindern (fehlende Produktstrategie, Techniklastigkeit, mangelndes Änderungs- und Risikomanagement, ...).

\section{Prozessbereiche eines kontinuierlichen Anforderungsmanagements}

Nachfolgend werden die Aufgaben der drei vorgestellten Prozessbereiche eines kontinuierlichen Anforderungsmanagement detailliert beschrieben. Wie in Abbildung 2 dargestellt, sind diese drei Prozessbereiche grundsätzlich eingegliedert in die umfassendere Organisation eines Kunden(beziehungs)management, eines Produktmanagements und eines Projektmanagements, wobei der Gesamtprozess des Anforderungsmanagements quer zu diesen funktionalen Organisationseinheiten liegt bzw. diese hinsichtlich des Umgangs mit Anforderungen verbindet.

\section{Kunden-Anforderungsmanagement}

Kundenorientierung wird zunehmend ein zentrales Leitbild für Organisationen. Im Mittelpunkt des Kunden(beziehungs)managements (customer relationship management CRM) steht die effiziente Verknüpfung von Marketing-, Vertriebs- und Serviceprozessen. Hauptziele sind die Identifizierung von Zielgruppen, die effektive Ansprache potentieller Kunden und die Maximierung der Kundenloyalität durch Steigerung der Kundenzufriedenheit.

Das Kunden-AM ist ein zentraler Teilprozess der Serviceprozesse im Kundenmanagement. Die Zufriedenstellung von Kunden beruht letztlich auf der Identifikation sowie der Erfüllung ihrer Bedürfnisse und Anforderungen. Aus Sicht des Kundenmanagements ist das Kunden-AM deshalb ein wesentliches Instrument zur langfristigen Kundenbindung, mit drei zentralen Aufgaben:

- Kundenanforderungen ermitteln. Bewertung und Beauftragung (Weiterleitung) erhobener oder gestellter Anforderungen von Kunden.

- Kundenprozesse aufnehmen. Antizipative Kundenbedürfnisanalyse durch Erstellung und Integration von Kundenprozessen.

- Geschäftsprozesse erstellen. Entwicklung und Abgleich von Geschäftsprozessmodellen auf der Grundlage eines Geschäftsmodells. 
Die Entwicklung von Geschäftsprozessmodellen dient dazu, die strategischen Zielsetzungen des Geschäftsmodells zu operationalisieren und daraus Anforderungen für notwendige unterstützende IT-Systeme abzuleiten (vgl. dazu das Ebenenmodell des Business Engineering nach [Österle95]). In der Sparkassen-Finanzgruppe umfasst dieses Geschäftsprozessmodell beispielsweise inzwischen ca. 180 Geschäftsprozesse für die Bereiche Finanzdienstleistungsfiliale, Vermögensmanagement und Immobilienzentrum.

Diese Geschäftsprozesse beschreiben die Soll-Geschäftsabläufe innerhalb einer Sparkasse; bspw. wie eine Inlandsüberweisung, eine Lastschrifteinreichung oder ein Kreditvertrag abgewickelt werden kann. Ein Kundenprozess ist demgegenüber „die Zusammenfassung aller Aufgaben, die der Kunde im Zusammenhang mit der uns gelieferten Ressource (Information, Dienstleistung und Produkt) zu erfüllen hat" [Österle00, S. 24]. Die Erstellung von Kundenprozessen und ihre Integration in die Geschäftsprozesse dient dazu, vorausschauend Kundenbedürfnisse aufzunehmen und sich damit gegenüber dem Kunden als umfassender Lösungsanbieter für einen Kundenprozess, etwa über ein Prozessportal, zu positionieren.

Betrachtet man beispielsweise den Kundenprozess „Immobilienerwerb“, so besteht dieser Prozess aus Kundensicht aus Schritten wie „über Angebote informieren“, „Finanzierung planen“, „Objekt aussuchen“, „Finanzierung“, „Umzug“, „Einrichtung“. Ein Finanzdienstleister muss versuchen, die Anforderungen dieses Prozesses aus Kundensicht zu verstehen und ihn gesamthaft durch Dienstleistungen und Produkte zu unterstützen - von der Ansparung über die Immobilienberatung der Finanzierung bis zur Lebensversicherung - um somit letztlich Cross-Selling-Potenziale zu nutzen. Beispiele für eine Immobilienerwerb-Portal-Unterstützung von Kundenprozessen durch Finanzdienstleister, wie etwa das Prozessportal der Credit Suisse www.yourhome.ch oder die Lösung der Hypovereinsbank unter www.immoseek.de.

Die dritte, eher klassische Aufgabe des Kunden-AM ist die Aufnahme, Bewertung und Beauftragung von Kundenanforderungen. Die folgende Abbildung 3 stellt diesen Prozess überblicksartig dar. Die Kundenmanager oder -betreuer nehmen Rohanforderungen des Kunden auf und konsolidieren diese. Rohanforderungen stellen Probleme bzw. Bedürfnisse dar, für welche eine Lösung gewünscht wird. Sie können im Rahmen von Workshops oder Interviews ermittelt oder direkt vom Kunden über ein Helpdesk oder ein AM-Werkzeug eingestellt werden. Eine Rohanforderung wird im Kunden-AM gemeinsam mit dem Kunden präzisiert und in eine standardisierte Kundenanforderung überführt. Zudem wird über die Wichtigkeit der Realisierung der Anforderung entschieden und gegebenenfalls ihre Umsetzung kontrolliert. Abhängig vom Typ der Anforderung (bspw. rechtliche, geschäftspolitische oder DV-Anforderung) können verschiedene Prozessvarianten definiert werden. 


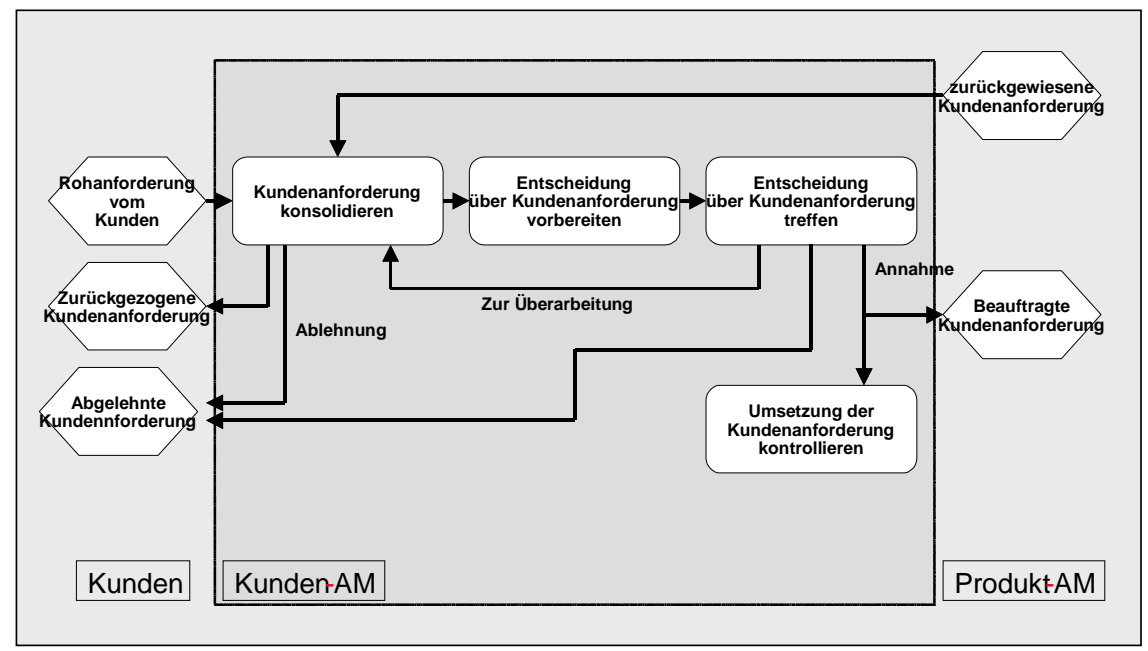

Abbildung 3: Bewertung und Beauftragung von Kunden-Anforderungen

\section{Produkt-Anforderungsmanagement}

Das Produkt-AM ordnet Kundenanforderungen im Hinblick auf die Weiter- oder Neuentwicklung ihrer Produkte bzw. Produktfamilien und der damit verbundenen IT-Strategie ein. Hauptaufgaben des Produkt-AM sind:

- Ableitung von Produktanforderungen. Bewertung und Bündelung von Kundenanforderungen, Überführung in Produktanforderungen und Schneidung von Produkten bzw. Anwendungen.

- Durchführung von Markt- und Konkurrenzanalysen. Diese Analysen dienen insbesondere für die Definition von Produktanforderungen bzgl. Leistungs- und Begeisterungsfaktoren.

- (Weiter)Entwicklung der Anwendungslandschaft. Die Anwendungslandschaft unterstützt die Schneidung von Produkten und die Festlegung der Produktfamilien und Produktlinien.

Hauptergebnis der Aktivitäten im Produkt-AM ist das Lastenheft als Ergebnisdokument der Produktplanungsphase. Im Lastenheft erfolgt eine bewusste Konzentration auf die fundamentalen Produktanforderungen. Dabei wird auf die präzise Formulierung des (zukünftigen) Produktumfeldes und der notwendigen Produkteigenschaften in diesem Umfeld fokussiert.

Die zweite und dritte Hauptaufgabe sind als Unterstützungsmaßnahmen für die Schneidung von Produkten aufzufassen. Die Anwendungslandschaft unterstützt die Ableitung von Produktanforderungen aus zugeordneten Kundenanforderun- 
gen, ermöglicht die Abgrenzung der fachlichen Architekturen und unterstützt die Definition von Systemarchitekturen (bspw. welche Architekturmuster sind $\mathrm{zu}$ verwenden? Woraus besteht der Produktkern? usw.).

Neben direkten Kundenanforderungen geben Markt- und Konkurrenzanalysen wichtige Hinweise zur Gestaltung von Produkteneigenschaften. Kano unterscheidet hierbei drei Arten von Anforderungen. Standard-oder Basisfaktoren stellen grundlegende Anforderungen an ein Produkt dar. Ohne die Erfüllung dieser Anforderungen kann das Produkt seinen Zweck für den Nutzer nicht erfüllen. Das Fehlen dieser Eigenschaften führt zu einer Ablehnung des Produktes (Beispiel Handy: die Möglichkeit, verschiedene Telefonnummern zu speichern). Bei Leistungsfaktoren wächst die Zufriedenheit mit dem Produkt proportional mit dem Erfüllungsgrad von Anforderungen (Handy: Mehrnetzfähigkeit). Sind Begeisterungsfaktoren erfüllt, führt dies zu einer sehr großen Zufriedenheit bei Kunden. Fehlen diese Faktoren, ist der Kunde aber nicht unbedingt unzufrieden (Handy: Internetzugang mit WAP-Technologie).

Die Herausforderung für das Produkt-AM liegt nun darin, die optimale Balance zwischen diesen Arten von Anforderungen zu finden. Sollen etwa neue attraktive Märkte gewonnen werden, muss besonders auf die Begeisterungsfaktoren geachtet werden. Allerdings können diese Begeisterungsfaktoren im Rahmen der Produktevolution sehr schnell zu Basisfaktoren migrieren, wie etwa auch an der Entwicklung der grafischen Benutzungsoberflächen für PCs deutlich wird.

\section{Projekt-Anforderungsmanagement}

Das Projekt-AM ist für die effiziente Realisierung und die damit verbundene Detaillierung der Produktanforderungen unter Einhaltung der zur Verfügung gestellten Ressourcen im Rahmen der Anwendungsentwicklung verantwortlich. Hierzu werden die Produktanforderungen des Lastenhefts detailliert und ein sog. Pflichtenheft oder Grobkonzept erstellt. Insbesondere werden dabei alle im Lastenheft identifizierten Anwendungsfälle vollständig und ausführlich mit allen Ausnahmen und Varianten beschrieben und ein erstes grobes Fachmodell (Klassenmodell bzw. Daten- und Funktionsmodell) erstellt.

Der Fokus im Pflichtenheft liegt - anders als im Lastenheft - nicht mehr auf dem Umfeld, den Kundenbedürfnissen und Rahmenbedingungen, sondern auf der präzisen Darstellung der gewünschten, im Projekt zu realisierenden Produkteigenschaften. Stellt das Lastenheft insbesondere das fachliche Planungsergebnis des Produktmanagements bzw. des Auftraggebers dar, so dient das Pflichtenheft mit seiner detaillierten Beschreibung aller Produktanforderungen als Grundlage für eine folgende Make-or-Buy-Entscheidung und für die Priorisierung und Bildung von Inkrementen. 
Da die meiste Literatur zum Requirements Engineering das Projekt-AM ausführlich behandelt, wird dieser Aspekt an dieser Stelle nicht weiter vertieft. Es sei jedoch darauf hingewiesen, dass das kontinuierliche Anforderungsmanagement mit leichtgewichteten Entwicklungsmodellen wie Extreme Programming [Beck99] oder Crystal Clear [Cockburn02] harmoniert. Diese Modelle verfolgen das Ziel, den Entwicklungsprozess selber flexibel und robust gegenüber Änderungen und fehlerhaften Anforderungen zu halten, indem beispielsweise die Anzahl der Ergebnistypen und -dokumente radikal reduziert wird, ein stark iteratives Vorgehen in sehr kleinen Schritten vorfolgt wird und kooperativ in kleinen Teams entwickelt wird.

Sicherlich werden bei komplexen Produktentwicklungen weiterhin schwergewichtige Vorgehensweisen mit langen Projektlaufzeiten und umfangreichen Teams notwendig sein. Da leichtgewichtige Entwicklungsprozesse aber weniger risikobehaftet sind und dem Kunden schneller einen Nutzen im Sinne einer lauffähigen Anwendung bringen, wird diese Form der Anwendungsentwicklung zunehmend eine wichtige Alternative. Hier hilft das Anforderungsmanagement, kleinere Projekte mit definiertem Umfang und Zielen zu schneiden sowie das Risiko eines scope creeping, d.h. einer Verschiebung des Projektfokus, zu vermindern.

\section{Adaption und Einführung}

Das im vorigen Abschnitt beschriebene Vorgehen muss natürlich an die Organisation bzw. dem Anwendungskontext angepasst werden. Hierdurch werden optimierte Prozesse sowie nachhaltige Prozessverbesserungen erst ermöglicht. Sowohl die Einführung als auch die kontinuierliche Prozessverbesserung sollte sich dabei am aktuellen Reifegrad des Anforderungsmanagements in einer Organisation orientieren (vgl. etwa [Sawyer97]). Nachdem in einem Assessment die Qualität der aktuellen Prozesse bewertet wurde, sind geeignete Verbesserungsmaßnahmen auszuwählen und die Sollablauf- und -aufbauorganisation des Anforderungsmanagements festzulegen. Dabei kann das im vorigen Kapitel beschriebene Vorgehen im Sinne eines Referenzmodells genutzt und adaptiert werden.

Im ersten Schritt sollten die Sollprozesse des Anforderungsmanagements soweit möglich durchgängig beschrieben werden. Dies gilt auch, falls bereits feststeht, dass bestimmte Aufgaben durch externe Organisationen durchgeführt werden, etwa das Projekt-AM durch einen selbständigen Auftragnehmer. Die durchgängige Betrachtung stellt sicher, dass die AM-Prozesse über Organisationsgrenzen hinweg optimiert und mögliche Schnittstellenprobleme minimiert werden.

Bei der Entwicklung der Sollprozesse werden Unterschiede in der Anzahl der Produkte und Kunden, den Produktarten, den Produktlebenszyklen und den am Prozess beteiligten Organisationen zu unterschiedlichen Prozessvarianten führen. 
Hinsichtlich der Produktarten sind beispielsweise unterschiedliche Abläufe für Standardprodukte, Produktfamilien oder Individualprodukte sinnvoll. Standardprodukte erfordern umfangreiche Kundenbedürfnis-, Konkurrenz- und Marktanalysen, wobei insbesondere auf Anforderungen zu Leistungs- und Begeisterungsfaktoren zu achten ist. Bei Produktfamilien ist eine enge Abstimmung der Anforderungen zum Produktkern und zu nichtfunktionalen Anforderungen wie Stabilität oder Erweiterbarkeit erforderlich. Für Individualprodukte sind schnelle Entwicklungszyklen und kurze Durchlaufzeiten der Anforderungen anzustreben.

Der Prozess des Anforderungs- und Änderungsmanagements kann für bewährte (Alt)Anwendungen stärker formalisiert und institutionalisiert werden, als für innovative Neuentwicklungen etwa im Bereich E-Business. Für eine Home-Banking Anwendung mit vielen Nutzern muss ein anderes AM-Verfahren vorgesehen werden, als etwa für eine Anwendung im Bereich von Abwicklungssystemen für den Zahlungsverkehr (Buchungs- oder Clearingsysteme) mit wenigen, sehr sachverständigen (Instituts-)Kunden. Um die Prozesskomplexität zu verringern und Kapazitäten von Stellen besser auszulasten, wird bei der folgenden Ableitung von Organisationseinheiten und der Stellenbildung allerdings häufig eine Konsolidierung erforderlich sein, falls in dieser Sollmodellierung zu viele Prozessvarianten erstellt wurden.

Ausgehend von den Abläufen und den dabei definierten Rollen kann anschließend die Bildung und Zuordnung von Organisationseinheiten und Stellen erfolgen (vgl. zum Vorgehen bei der prozessorientierten Gestaltung einer Aufbauorganisation [Kugeler00]). Abbildung 4 skizziert beispielhaft die Adaption des im vorigen Abschnitts beschriebenen Vorgehens für die Bildung von Organisationseinheiten ausgehend von ermittelten Prozesseigenschaften. Links sind die Prozessbereiche Kunden-, Produkt- und Projekt-AM und mögliche Zuordnungen von Organisationseinheiten im Sinne von Verantwortungsbereichen dargestellt. Die Kreise symbolisieren die durchzuführenden Aufgaben im Rahmen des Anforderungsmanagements. Die umgebenden Rechtecke/Quadrate stellen Organisationseinheiten mit Auftraggeber-/Auftragnehmerrollen dar. In den weiteren Spalten sind diese Organisationsbereiche als Rollen beispielhaft benannt und Eigenschaften angegeben, welche die Aufteilung empfehlenswert machen.

Die erste Reihe zeigt beispielsweise eine gemäß den AM-Prozessbereichen gegliederte Aufteilung mit einem eigenständigen Kunden-, Produkt und Projektmanagement. Diese Aufteilung ist sinnvoll, wenn viele Kunden, viele Produkte und viele Projekte zu betreuen sind. Sie ist typisch für Rechenzentren oder selbständige IT-Dienstleister.

Ein exemplarisches Beispiel für diese Aufteilung des Anforderungsmanagements sind die Verbandsrechenzentren der Sparkassen-Finanzgruppe. Diese fungieren als IT-Dienstleister für angeschlossene Sparkassen mit einer Vielzahl von IT-Produkten und Entwicklungsprojekten. In [Kräft99] wird diese Form der Aufteilung 
für das Verbandsrechenzentrum dvg Hannover der Sparkassen-Finanzgruppe beschrieben.

Die Definition der Rollen und die Beziehungen zwischen den Organisationseinheiten sollte auf verbindlichen Auftraggeber/Auftragnehmer-Regelungen und Budgetverantwortlichkeiten beruhen. Soll das Kunden-AM als Auftraggeber gegenüber dem Produkt-AM operieren oder soll das Kunden-AM und das ProjektAM vom Produkt-AM beauftragt werden?
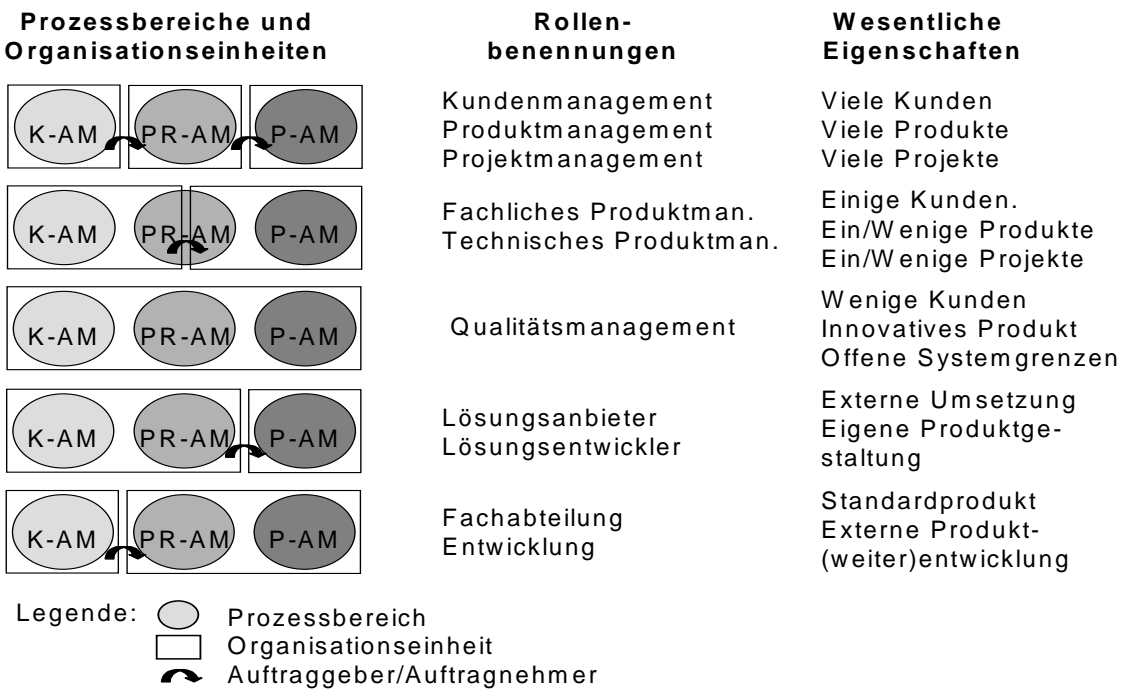

Abbildung 4: Adaptionen des Vorgehens im Anforderungsmanagement

Die erste Variante kommt beispielsweise für Rechenzentren oder IT-Abteilungen in Frage, die auftragsgebunden IT-Dienstleistungen für Fachabteilungen erstellen und anbieten. Ein anderes Vorgehen ist zu wählen, falls das Produktmanagement als Auftraggeber eines Kunden-AM und eines Projekt-AM fungiert. In dieser klassischen, marktorientierten Variante verfügt das Produktmanagement über das Produktbudget. Es hat gegenüber dem Management die betriebswirtschaftliche Verantwortung für den Absatz und die Weiterentwicklung eines Standardproduktes. Nachdem die Organisationseinheiten aus den Prozessen abgeleitet und geschnitten wurden, werden die identifizierten Rollen mit dem Ziel ganzheitlicher Aufgabenstrukturen zu Stellen kombiniert. Diese Stellenbildung für die Prozesseigner und Prozessteams erfolgt mit Blick auf die Anforderungen an Kapazitätsauslastung, Kenntnissen und Fähigkeiten der zukünftigen Stelleninhaber.

Beispielsweise werden im SIZ die Aufgaben des Kunden-AM und Produkt-AM zusammengefasst, beim externen Auftragnehmer sind zwei Stellen für das Produkt- und das Projekt-AM vorgesehen. Das Kunden-AM im SIZ erhält seine Anforderungen vom Produkt-AM eines Verbandrechenzentrums und einer 
Landesbank bzw. dem Kunden-AM eines Verbundpartners. Innerhalb von (Groß)Sparkassen können sich wiederum verschiedene Prozessbereiche des Anforderungsmanagements befinden, wobei diese ihre Anforderungen beispielsweise über einen Verband, welcher die Rolle des Kunden-AM ausübt, an ein Verbandsrechenzentrum melden. Während in vielen Organisationen eine Stelle für das Anforderungsmanagement ausreichend ist, müssen diese Aufgaben im Rechenzentrum aufgrund der großen Anzahl an Projekten und Produkten auf mehrere Stellen verteilt werden. Ähnlich sind im Verband mehrere Stellen für das Kunden-AM zuständig, da eine Vielzahl von Sparkassen Anforderungen an die IT stellen können und als Kunden zu betreuen sind.

Nachdem Organisationseinheiten und Stellen mit operativen Aufgaben gebildet wurden, sind diese unterhalb der Geschäftsführung in ein Leitungssystem nach einheitlichen Verantwortungsbereichen einzuordnen. Als letzter Schritt vor dem Roll-Out der Prozesse wird die vorgenommene Stellenbildung dahingehend überprüft, ob die Stellen ausgelastet sind und die Prozessschnittstellen bzw. die festgelegten Abläufe noch lokal optimiert werden können (vgl. [Kugeler00]).

Die Einführung der neugestalteten AM-Prozesse kann nach unterschiedlichen Strategien erfolgen: Schrittweise Einführung (Step-by-Step), pilotierte Einführung oder Organisationsweit gleichzeitige Einführung (Big-Bang). Eine Big-Bang-Lösung strebt primär eine schnelle Umsetzung der Organisations- und Prozessverbesserung an, während eine stufenweise Einführung den Sicherheitsaspekt stärker gewichtet.

Welche der dargestellten Strategien für die Einführung oder Weiterentwicklung des Anforderungsmanagements zu wählen ist, hängt stark von der konkreten Situation ab. Falls der Leidensdruck und die Unzufriedenheit der Kunden dies nicht erfordert, sollte grundsätzlich eine pilotierte Einführung trotz der längeren Einführungszeiten und den höheren Einführungskosten bevorzugt werden, um organisationsspezifische Erfahrungen zu gewinnen, Prozesse weiter optimieren zu können und insbesondere das Risiko von Produktivabnahmen zu reduzieren.

\section{Ausblick}

Die Notwendigkeit eines umfassenden Ansatzes für das Anforderungsmanagement basiert auf der Einsicht, dass die Ursachen für viele Probleme beim Umgang mit Anforderungen in Entwicklungsprojekten gar nicht in den Projekten selber zu finden sind. Unrealistische oder mangelnde Projektplanung im Produktmanagement, etwa bzgl. Fertigstellungsterminen, kann in einem Projekt zwar teilweise aufgefangen werden, etwa durch Priorisierung der Anforderungen oder inkrementelle Systementwicklung, zufriedene Kunden und eine längerfristige Kunden- 
bindung ist damit aber nicht zu erreichen (von der Zufriedenheit der Projektmitarbeiter ganz zu schweigen).

Eine wirkliche Verbesserung wird nur dann erreicht, wenn der gesamte Prozess des Anforderungsmanagements ausgehend von der Erhebung einer Kundenanforderung und Beauftragung bis zur Lösungsbereitstellung und anschließenden Kundenbetreuung optimiert und als permanenter Prozess verankert wird.

Ein solches Anforderungsmanagement kann dann auch die notwendige Brücke zwischen dem Business Engineering für die systematische Gestaltung des Geschäfts und dem Software Engineering für die systematische Gestaltung der Anwendungen bilden. Das vom Business Engineering verfolgte Ziel, das Geschäft und die abgeleiteten Geschäftsprozesse von Unternehmen systematisch zu strukturieren und an Veränderungen in relevanten Umweltsegmenten rasch anzupassen, bedingt eine ständige Weiterentwicklung der unterstützenden Informationstechnologie und somit ein kontinuierliches Anforderungsmanagement. Die angestrebte Konvergenz von Geschäftsprozessen und Informationstechnologie ist daher ohne ein kontinuierliches Anforderungsmanagement nicht erreichbar.

\section{Literatur}

[Beck99] Beck, K.: Extreme Programming Explained: Embrace Change. Addison Wesley 1999

[Cockburn02] Cockburn, A.: Crystal Clear: A Human-Powered Methodology for Small Teams. Addison-Wesley (erscheint voraussichtlich 2002; Online-Version siehe members.aol.com/humansandt/crystal/clear)

[Davis93] Davis, A.M.: Software Requirements. Objects, Functions, \& States. Prentice Hall 1993

[ESPITI95] European Software Process Improvement Traning Initiative (Hrsg.): User Survey Report. ESPITI 1995

[Gause93] Gause, D.C.; Weinberg, G.M.: Software Requirements. Anforderungen erkennen, verstehen und erfüllen. Hanser 1993

[Kräft99] Kräft, D.; Fabian, D.: S-INI-Prozess: Von der Idee zur Anwendung. In: update 3/99, S. 21-23

[Kugeler00] Kugeler, M.; Vieting, M.: Gestaltung einer prozessorientiert(er)en Aufbauorganisation. In: Becker, J.; Kugeler, M.; Rosemann, M. (Hrsg.): Prozessmanagement. Springer 2000, S. 187-232

[Leffingwel199] Leffingwell, D.; Widrig, D.: Requirements Management. A Unified Approach. Addison-Wesley 1999 
[Martin96] Martin, J.N.; Hahill, A.T. (Hrsg.): Systems Engineering Guidebook: A Process for Developing Systems and Products. Prentice Hall 1996

[Österle95] Österle, H.: Business Engineering, Prozess- und Systementwicklung, Band 1: Entwurfstechniken. Springer 1995

[Österle00] Österle, H.: Business Engineering, Auf dem Weg zum Unternehmen des Informationszeitalters. Springer 2000

[Poh196] Pohl, K.: Process-Centered Requirements Engineering. Wiley 1996

[Poh199] Pohl, K.: Continuous Documentation of Information System Requirements. Habilitation, RWTH Aachen 1999

[Robertson99] Robertson S., Robertson J.: Mastering the Requirements Process. Addison Wesley 1999

[Rupp01] Rupp, C.: Requirements Engineering und -Management. Hanser 2001

[Sawyer97] Sawyer, P., Sommerville, I.; Viller, S.: Requirements Process Improvement Through the Phased Introduction of Good Practice. In: Software Process - Improvement and Practice 1 (1997) 3, S. 19-34

[Simon85] Simon, H. A.: The Sciences of the Artificial. 2. Auflage. MIT Press 1985

[Standish95] Standish Group (Hrsg.): The Scope of Software Development Project Failures. CHAOS-Report. Standish Group 1995

[Schienmann00a] Schienmann, B.; Strassenburg, S.; Schelling, B.: Erfolgreiches Anforderungsmanagement. In: Betriebswirtschaftliche Blätter 49 (2000) 12, S. 597-602

[Schienmann00b] Schienmann, B.: Anforderungsmanagement in der Sparkassenorganisation. In:. Mayr, H.C.; Hesse, W.; Oberweis, A.; Kop, Ch. (Hrsg.): Software-Management 2000. Proc. Software Management'2000. Bd. 149. Wien: OCG 2000, S. 133-149

[Stevens98] Stevens, R.; Brook, P.; Jackson, K.; Arnold, S:: Systems Engineering. Coping with Complexity. Prentice Hall 1998

[Wiegers99] Wiegers, K.E.: Software Requirements. Microsoft Press 1999 